\title{
A simple model for lamellar peritectic coupled growth with peritectic reaction
}

\author{
LUO LiangShun ${ }^{\dagger}$, SU YanQing, GUO JingJie, LI XinZhong \& FU HengZhi \\ School of Materials Science and Engineering, Harbin Institute of Technology, Harbin 150001, China
}

The lamellar peritectic coupled growth in $\mathrm{Fe}-\mathrm{Ni}$ peritectic system was investigated using the equilibrium Boettinger-Jackson-Hunt model. It was found that the slope of the undercooling vs. lamellar spacing is very near zero around the minimum overheating, and the coupled growth can exist under this condition even if the slope of the undercooling vs. lamellar spacing curve is slightly smaller than zero. In addition, the peritectic reaction can never reach completion during the peritectic coupled growth. So the equilibrium peritectic coupled growth was modified by considering the incompletion of the peritectic reaction. It was shown that when the fractions of the peritectic reaction reach $60 \%-80 \%$ completion, the calculated undercooling vs. lamellar spacing curves agree well with the experimental observations in the directionally solidified Fe-Ni alloys.

peritectic alloys, coupled growth, directional solidification, peritectic reaction

When the binary eutectic alloys are performed at a proper growth velocity $V$ under a sufficiently strong thermal gradient $G$ to solidify directionally, periodic stationary patterns consisting of planar lamellar of the crystal phases in the direction parallel to the growth direction usually form. This is called the diffusion controlled eutectic coupled growth ${ }^{[1]}$. The coupled growth is the result of solutal diffusion in the liquid and capillary forces at interface. Eutectic coupled growth (ECG) has been extensively investigated because the interface evolution of ECG is a typical nonlinear process and the in situ composites which can be produced by ECG have potential applications in some specific fields ${ }^{[2-5]}$.

Most of the researches related to ECG are based on the analysis of eutectic solidifications given by Jackson and Hunt ${ }^{[1]}$. Recently, peritectic solidification has received much attention because many new advanced materials have peritectic reaction during solidification, such as potential high temperature structure materials Ti-Al, Ni-Al, permanent magnetic materials $\mathrm{Nd}-\mathrm{Fe}-\mathrm{B}$, and high temperature superconductors $\mathrm{Y}-\mathrm{Ba}-\mathrm{Cu}-\mathrm{O}$, etc. ${ }^{[6]}$. The possibility of eutectic-like coupled growth in

Received February 11, 2007; accepted May 18, 2007 doi: $10.1007 / \mathrm{s} 11433-007-0042-\mathrm{x}$

'Corresponding author (email: luols80@gmail.com)

Supported by the National Natural Science Foundation of China (Grant Nos. 50395102 and 50271020) and the Program for New Century Excellent Talents in University (Grant No. 05-0530) 
peritectic systems is one important topic. Boettinger ${ }^{[7]}$ examined peritectic coupled growth (PCG) using the eutectic theory developed by Jackson and Hunt ${ }^{[1]}$, and found that the coupled growth in peritectic systems is unlikely on theoretical grounds in 1974. However, PCG has been observed in many peritectic systems since $1994^{[8-11]}$. Figure 1 shows the coupled growth in directionally solidified Fe-Ni alloys. Even so, many basic aspects of PCG, such as the conflict between the existence of PCG with theoretical predictions and the basic characteristics of PCG, are still largely unclear, in spite of many valuable experimental and numerical investigations ${ }^{[12]}$. In addition, the peritectic reaction has significant influences on PCG, which has not been taken into account in the peritectic coupled growth model. In this paper, we present a simple model of PCG considering the influences of the peritectic reaction, especially the influence of the peritectic reaction on the volume

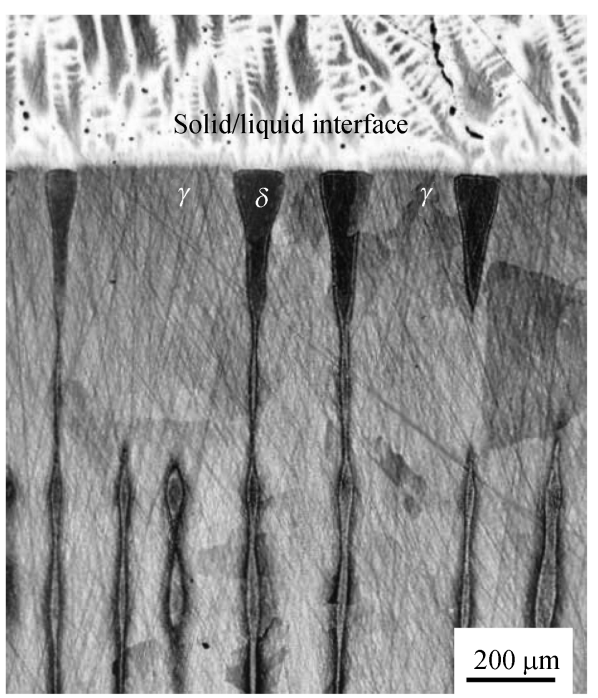

Figure 1 Coupled growth obtained in directional solidification of Fe-4.5Ni alloy. $G=12 \mathrm{~K} / \mathrm{mm} ; V=5$ $\mu \mathrm{m} / \mathrm{s}$. fractions of two solid phases.

\section{Equilibrium peritectic coupled growth model}

The diffusion equation for PCG should be solved at first to quantitatively model the coupled growth behavior in peritectic systems. Consider a near planar interface of primary phase $\alpha$ and peritectic phase $\beta$, with $S_{a}$ and $S_{\beta}$ being respectively the half width of each phase, as shown in Figure 2. During PCG, the solute B atoms diffuse from liquid/ $\alpha$ interface to liquid $/ \beta$ interface, and the solvent $\mathrm{A}$ atoms diffuse from liquid $/ \beta$ interface to liquid/ $\alpha$ interface. For the steady state coupled growth with the coordinate system shown in Figure 2, the diffusion equation of PCG becomes

$$
\nabla^{2} C_{1}+\frac{V}{D} \frac{\partial C_{1}}{\partial z}=0
$$

where $C_{1}$ is the liquid composition at the interface, and $D$ is the diffusion coefficient. For the rod coupled growth, eq. (1) is in a three-dimensional form; whereas for the lamellar coupled growth, eq. (1) is in a two-dimensional form as

$$
\frac{\partial^{2} C_{1}}{\partial x^{2}}+\frac{\partial^{2} C_{1}}{\partial z^{2}}+\frac{V}{D} \frac{\partial C_{1}}{\partial z}=0
$$

To solve eq. (1), some assumptions should be made.

(1) The solid/liquid interface is plane. This is obviously incorrect because the interface has a greater curvature when the $\alpha / \beta$ interface is approached to make the trijunctions mechanics balanced. However, this assumption makes the problem easy to solve. Besides, it is reasonable to treat the nonplanar interface as perturbations for the regular eutectic coupled growth ${ }^{[13]}$.

(2) The composition of the liquid far from the interface is the initial composition $C_{0}$, i.e. $z \rightarrow \infty$, $C_{1} \rightarrow C_{0}$.

(3) Because of the symmetry of the lamellar coupled growth, a solution is necessary only for the 
region $0 \leqslant x<S_{\alpha}+S_{\beta}$ and $\partial C_{1} / \partial x=0$ at $x=0$ and $S_{\alpha}+S_{\beta}$.

(4) For the steady state growth, the mass conservation requires

$$
\begin{gathered}
\left.\frac{\partial C_{1}}{\partial z}\right|_{z=0}=-\frac{V}{D}\left(C_{0}-C_{\alpha}\right), \quad 0 \leqslant x \leqslant S_{\alpha}, \\
\left.\frac{\partial C_{1}}{\partial z}\right|_{z=0}=-\frac{V}{D}\left(C_{0}-C_{\beta}\right), \quad S_{\alpha}<x<S_{\beta}+S_{\alpha} .
\end{gathered}
$$

(5) Peritectic reaction reaches completion during PCG, and then the volume fractions of peritectic two-phase can be calculated using the equilibrium phase diagram.
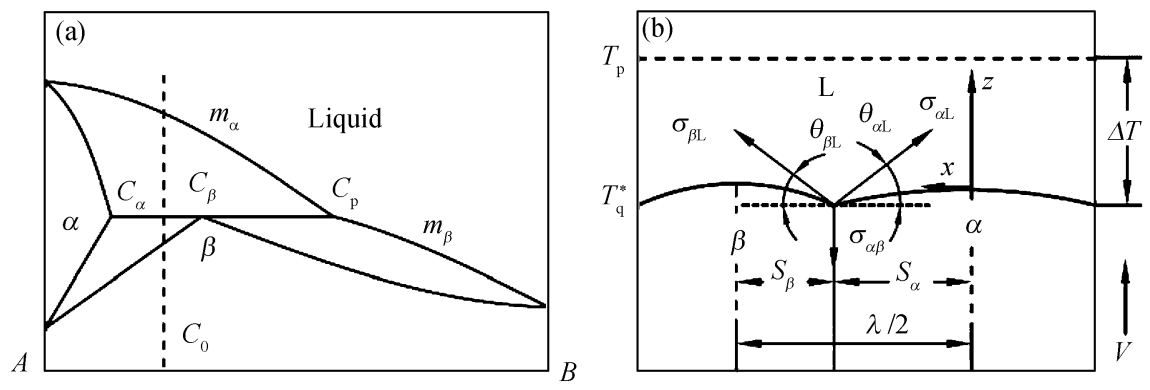

Figure 2 Schematic representations of peritectic phase diagram (a) and two-phase lamellar peritectic coupled growth (b) showing the definitions of the parameters used in this work.

Using the above assumptions and boundary conditions, the solution of diffusion equation (2) is ${ }^{[7]}$

$$
C_{1}(x, z)=C_{0}+B_{0} \exp \left(-\frac{V}{D} z\right)+\sum_{n=1}^{\infty} B_{n} \cos \left(\frac{n \pi x}{S_{\alpha}+S_{\beta}}\right) \exp \left(\frac{-n \pi}{S_{\alpha}+S_{\beta}} z\right) .
$$

The Fourier coefficients in eq. (4) are

$$
\begin{gathered}
B_{0}=C_{\mathrm{p}}-\frac{C_{\alpha} S_{\alpha}+C_{\beta} S_{\beta}}{S_{\alpha}+S_{\beta}}, \\
B_{n}=\frac{2}{(n \pi)^{2}}\left(S_{\alpha}+S_{\beta}\right) \frac{V}{D}\left(C_{\beta}-C_{\alpha}\right) \sin \left(\frac{n \pi S_{\alpha}}{S_{\alpha}+S_{\beta}}\right) .
\end{gathered}
$$

The solutions are valid when $\frac{\pi}{S_{\alpha}+S_{\beta}} \gg \frac{V}{2 D}$.

Similar to the treatment by Jackson and Hunt ${ }^{[1]}$, the average compositions in the liquid at the interface in front of the $\alpha$ and $\beta$ phases $(z=0)$ are

$$
\begin{gathered}
\bar{C}_{\alpha}=\frac{1}{S_{\alpha}} \int_{0}^{S_{\alpha}} C(x, 0) \mathrm{d} x=C_{0}+B_{0}+\frac{2\left(S_{\alpha}+S_{\beta}\right)^{2}}{S_{\alpha}} \frac{V}{D}\left(C_{\beta}-C_{\alpha}\right) P, \\
\bar{C}_{\beta}=\frac{1}{S_{\beta}} \int_{S_{\alpha}}^{S_{\alpha}+S_{\beta}} C(x, 0) \mathrm{d} x=C_{0}+B_{0}-\frac{2\left(S_{\alpha}+S_{\beta}\right)^{2}}{S_{\beta}} \frac{V}{D}\left(C_{\beta}-C_{\alpha}\right) P,
\end{gathered}
$$

where $P=\sum_{n=1}^{\infty}\left(\frac{1}{n \pi}\right)^{3} \sin ^{2}\left(\frac{n \pi S_{\alpha}}{S_{\alpha}+S_{\beta}}\right)$. 
During the coupled growth, the difference between the interface temperature $T^{*}$ and $T_{\mathrm{p}}$, i.e. the undercooling of PCG, is made up of three parts ${ }^{[1]}$

$$
\Delta T=\Delta T_{\mathrm{c}}+\Delta T_{r}+\Delta T_{\mathrm{k}},
$$

where $\Delta T_{\mathrm{c}}$ is diffusional undercooling, $\Delta T_{r}$ is curvature undercooling, and $\Delta T_{\mathrm{k}}$ is kinetic undercooling, which can usually be ignored in metallic binary systems ${ }^{[1]}$. The diffusional undercooling $\Delta T_{\mathrm{c}}$ comes from the departure of the local composition from peritectic composition $C_{\mathrm{p}}$, which can be written as

$$
\Delta T_{\mathrm{c}}=m\left(C_{\mathrm{p}}-C_{1}(x, z)\right)
$$

where $m$ is the liquidus slope.

The curvature undercooling can be obtained as ${ }^{[1]}$

$$
\Delta T_{r}=a_{i}^{\mathrm{L}} / r=\frac{\sin \theta}{S},
$$

where $a_{i}^{\mathrm{L}}$ is a constant given by the Gibbs-Thomson relationship, and $r$ is the local curvature of the interface.

The average undercooling at the interface for the lamellar peritectic coupled growth is then obtained by inserting the average composition from eq. (6) into eqs. (8) and (7) ${ }^{[7]}$ :

$$
\begin{aligned}
\Delta T_{\alpha} & =m_{\alpha}\left[C_{0}+B_{0}+\frac{2\left(S_{\alpha}+S_{\beta}\right)^{2}}{S_{\alpha}} \frac{V}{D}\left(C_{\beta}-C_{\alpha}\right) P\right]+\frac{a_{\alpha}^{\mathrm{L}}}{S_{\alpha}}, \\
\Delta T_{\beta} & =m_{\beta}\left[-C_{0}-B_{0}+\frac{2\left(S_{\alpha}+S_{\beta}\right)^{2}}{S_{\beta}} \frac{V}{D}\left(C_{\beta}-C_{\alpha}\right) P\right]+\frac{a_{\beta}^{\mathrm{L}}}{S_{\beta}},
\end{aligned}
$$

where

$$
\begin{aligned}
& a_{\alpha}^{\mathrm{L}}=\left(T_{\mathrm{p}} / L\right)_{\alpha} \sigma_{\alpha \mathrm{L}} \sin \theta_{\alpha \mathrm{L}}, \\
& a_{\beta}^{\mathrm{L}}=\left(T_{\mathrm{p}} / L\right)_{\beta} \sigma_{\beta \mathrm{L}} \sin \theta_{\beta \mathrm{L}},
\end{aligned}
$$

where $m_{\alpha}$ and $m_{\beta}$ are the liquidus slopes (defined as positive) of $\alpha$ and $\beta$ phases, $L_{\alpha}$ and $L_{\beta}$ are the latent heats per unit volume, $\sigma_{\alpha \mathrm{L}}$ and $\sigma_{\beta \mathrm{L}}$ are the solid/liquid surface energies per unit area, $\theta_{\alpha \mathrm{L}}$ and $\theta_{\beta \mathrm{L}}$ are contact angles of liquid/ $\alpha$ and liquid/ $\beta$ interfaces at the trijunctions, and $V$ is the growth velocity.

If $\lambda$ is defined to be the lamellar spacing $2\left(S_{\alpha}+S_{\beta}\right)$ and $\xi$ to be the ratio of the lamellar spacings of $\beta$ and $\alpha$ phases $S_{\beta} / S_{\alpha}$, then $S_{\alpha}$ and $S_{\beta}$ can be written as

$$
\begin{aligned}
& S_{\alpha}=\lambda /[2(1+\xi)], \\
& S_{\beta}=\lambda \xi /[2(1+\xi)] .
\end{aligned}
$$

Assuming the average undercooling of peritectic two-phase is equal, i.e. $\Delta T_{\alpha}=\Delta T_{\beta}=\Delta T$, the undercooling of the interface can be obtained by inserting eq. (12) into eq. (10) and combined with eq. (10) to eliminate $C_{0}+B_{0}$ as ${ }^{[7]}$

$$
\frac{\Delta T}{m}=Q^{\mathrm{L}} \lambda V+\frac{a^{\mathrm{L}}}{\lambda}
$$

where 


$$
\begin{gathered}
\frac{1}{m}=\frac{1}{m_{\alpha}}-\frac{1}{m_{\beta}}, \\
Q^{\mathrm{L}}=\frac{P(1+\xi)^{2}}{\xi D}\left(C_{\beta}-C_{\alpha}\right), \\
a^{\mathrm{L}}=2(1+\xi)\left(\frac{a_{\alpha}^{\mathrm{L}}}{m_{\alpha}}-\frac{a_{\beta}^{\mathrm{L}}}{\xi m_{\beta}}\right) .
\end{gathered}
$$

It can be seen from eq. (13) that the dependence of $\Delta T$ on $\lambda$ for the peritectic coupled growth has the same form as that for the eutectic coupled growth. However, $m$ in eq. (2a) is always negative because $m_{\alpha}>m_{\beta}$, which is contrary to the eutectic case. $Q^{\mathrm{L}}$ and $\lambda$ are always positive, therefore the dependence of $\Delta T$ on $\lambda$ at constant $V$ depends on the sign of $a^{\mathrm{L}[7]}$. It can have either sign, depending on the relative values of the surface energies and the ratio of the halfwidths of the two solid phases. As a result, there should be a critical composition $C^{*}$ leading to $a^{\mathrm{L}}=0$. Let $a^{\mathrm{L}}=0$ in eq. (14), one obtains

$$
C^{*}=\frac{C_{\beta}-C_{\alpha}}{C_{\mathrm{p}}-C_{\alpha}} C_{\mathrm{p}}+\frac{C_{\mathrm{p}}-C_{\beta}}{C_{\mathrm{p}}-C_{\alpha}} C_{\alpha}-\frac{C_{\beta}-C_{\alpha}}{1-\frac{L_{\beta}}{L_{\alpha}} \frac{\sigma_{\alpha \mathrm{L}}}{\sigma_{\beta \mathrm{L}}} \frac{m_{\alpha}}{m_{\beta}} \frac{\sin \theta_{\beta \mathrm{L}}}{\sin \theta_{\alpha \mathrm{L}}}} .
$$

\section{Results in Fe-Ni system and discussion}

The critical composition $C^{*}$ for Fe-Ni system is 4.2 at.\%Ni, which was calculated using eq. (15). The physical and material values used for calculation are listed in Table 1, defining $\alpha$ as primary phase $\delta$ ferrite and $\beta$ as peritectic phase $\gamma$ austenite in Fe-Ni system. When the composition of peritectic Fe-Ni alloy is greater than $4.20 \mathrm{Ni}, a^{\mathrm{L}}>0$, the undercooling of Fe-Ni PCG is negative regardless of $\lambda$; whereas the composition is lower than $4.20 \mathrm{Ni}, a^{\mathrm{L}}<0$, the undercooling decreases monotonically from positive to negative as $\lambda$ increases, as shown in Figure 3 . When the composition is $4.20 \mathrm{Ni}$, the $\Delta T-\lambda$ curve becomes a line.

The argument of Cahn presented by Jackson and Hunt states that the morphological stability of the coupled growth depends on the sign of slope of $\Delta T$ versus $\lambda^{[1]}$. Whenever $\mathrm{d} \Delta T / \mathrm{d} \lambda<0$, the

Table 1 Physical and material parameters of Fe-Ni alloy

\begin{tabular}{clll}
\hline Symbol & Values & \multicolumn{1}{c}{ Definition } & Ref. \\
\hline$T_{\mathrm{p}}$ & $1790.4 \mathrm{~K}$ & peritectic temperature & {$[12]$} \\
$C_{\alpha}$ & $3.83 \mathrm{at} \% \mathrm{Ni}$ & composition of $\alpha$ phase at $T_{\mathrm{p}}$ & {$[12]$} \\
$C_{\beta}$ & $4.33 \mathrm{at} . \% \mathrm{Ni}$ & composition of $\beta$ phase at $T_{\mathrm{p}}$ & {$[12]$} \\
$C_{\mathrm{p}}$ & $4.91 \mathrm{at} . \% \mathrm{Ni}$ & composition of liquid phase at $T_{\mathrm{p}}$ & {$[12]$} \\
$k_{\alpha}$ & 0.78 & partition coefficient of $\alpha$ & {$[12]$} \\
$k_{\beta}$ & 0.88 & partition coefficient of $\beta$ & {$[12]$} \\
$m_{\alpha}$ & $4.61 \mathrm{~K} / \mathrm{at} . \%$ & liquidus slope of $\alpha$ phase at $T_{\mathrm{p}}$ & {$[14]$} \\
$m_{\beta}$ & $2.16 \mathrm{~K} / \mathrm{at} . \%$ & liquidus slope of $\beta$ phase at $T_{\mathrm{p}}$ & {$[14]$} \\
$\Gamma_{\alpha}$ & $1.93 \times 10^{-7} \mathrm{~K} \cdot \mathrm{m}$ & Gibbs-Thomson coefficient of $\alpha$ phase $\Gamma_{\alpha}=T_{\mathrm{p}} \sigma_{\alpha \mathrm{L}} / L_{\alpha}$ & {$[15]$} \\
$\Gamma_{\beta}$ & $2.37 \times 10^{-7} \mathrm{~K} \cdot \mathrm{m}$ & Gibbs-Thomson coefficient of $\beta$ phase $\Gamma_{\beta}=T_{\mathrm{p}} \sigma_{\beta \mathrm{L}} / L_{\beta}$ & {$[15]$} \\
$\theta_{\alpha \mathrm{L}}$ & $50.5^{\circ}$ & angle of liquid $/ \alpha$ interface at trijunctions & {$[16]$} \\
$\theta_{\beta \mathrm{L}}$ & $61.9^{\circ}$ & angle of liquid $/ \beta$ interface at trijunctions & {$[14]$} \\
$D$ & $5.2 \times 10^{-9} \mathrm{~K} \cdot \mathrm{s} / \mathrm{m}^{2}$ & diffusion coefficient of Ni at $T_{\mathrm{p}}$ & \\
\hline
\end{tabular}




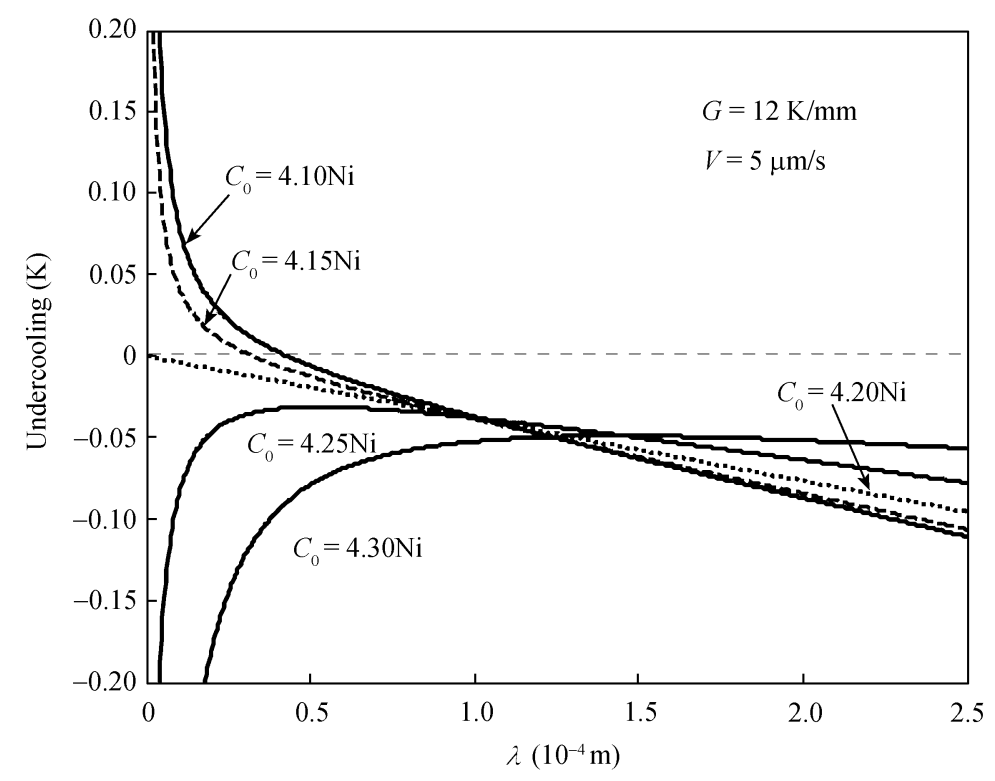

Figure 3 Variations of the interface undercooling with lamellar spacing in Fe-Ni alloys at $5 \mu \mathrm{m} / \mathrm{s}$.

perturbations of $\lambda$ will grow, and the coupled growth is unstable. Whenever $\mathrm{d} \Delta T / \mathrm{d} \lambda>0$, the perturbations of $\lambda$ will decay, and the coupled growth is stable to persist in time. When $a^{\mathrm{L}}<0$, $\mathrm{d} \Delta T / \mathrm{d} \lambda<0$ for all $\lambda$ and the peritectic coupled growth is unstable. When $a^{\mathrm{L}}>0, \Delta T$ is always negative regardless of $\lambda$ or $V$, and there is a minimum overheating. The $\mathrm{d} \Delta T / \mathrm{d} \lambda$ is positive when $\lambda$ is smaller than the minimum overheating, and is negative when $\lambda$ is greater than the minimum overheating, as shown in Figure 3.

Compared with ECG, the $\Delta T-\lambda$ curve of PCG has some specific characteristics.

(1) The composition has a more significant influence on the $\Delta T-\lambda$ curves of PCG. It can be seen from Figure 3 that when the composition just increases from $4.25 \mathrm{Ni}$ to $4.30 \mathrm{Ni}$, the minimum overheating will increase from $48 \mu \mathrm{m}$ to $156 \mu \mathrm{m}$. This may give one explanation to the irregular spacing of lamellar $\mathrm{PCG}^{[12]}$ that the segregation is often present in the directional solidification of peritectic alloys ${ }^{[11]}$.

(2) The slope of the $\Delta T-\lambda$ curve is near zero within a large of $\lambda$ around the minimum overheating, especially when $V$ is small, as shown in Figure 4. That is to say, the variation of the perturbations in the shape of the solid/liquid interface is very slow. Under this condition, the coupled growth can persist in time even if the slope of $\Delta T-\lambda$ curve is slightly below zero. This may be the reason that the coupled growth can exist when the slope of $\Delta T-\lambda$ curve is slightly below zero which was observed in the experiments and numerical simulations ${ }^{[12]}$.

In previous work ${ }^{[17]}$, we found that the peritectic reaction around liquid $/ \alpha / \beta$ trijunctions has significant influences on PCG. If the undercooling of the peritectic reaction is ignored, the interface of PCG is slightly above $T_{\mathrm{p}}$, which agrees well with the theoretical predictions ${ }^{[6,7,18]}$. The overheating of the interface in the coupled growth of directionally solidified Fe-4.3Ni alloy at $5 \mu \mathrm{m} / \mathrm{s}$ is about $0.02 \mathrm{~K}$; while in the theoretical calculation using eq. (13), the minimum overheating is about $0.05 \mathrm{~K}$, which is greater than the experimental observations. The difference may come from the errors in the physical and material parameters and the oversimplifications in the model. However, 

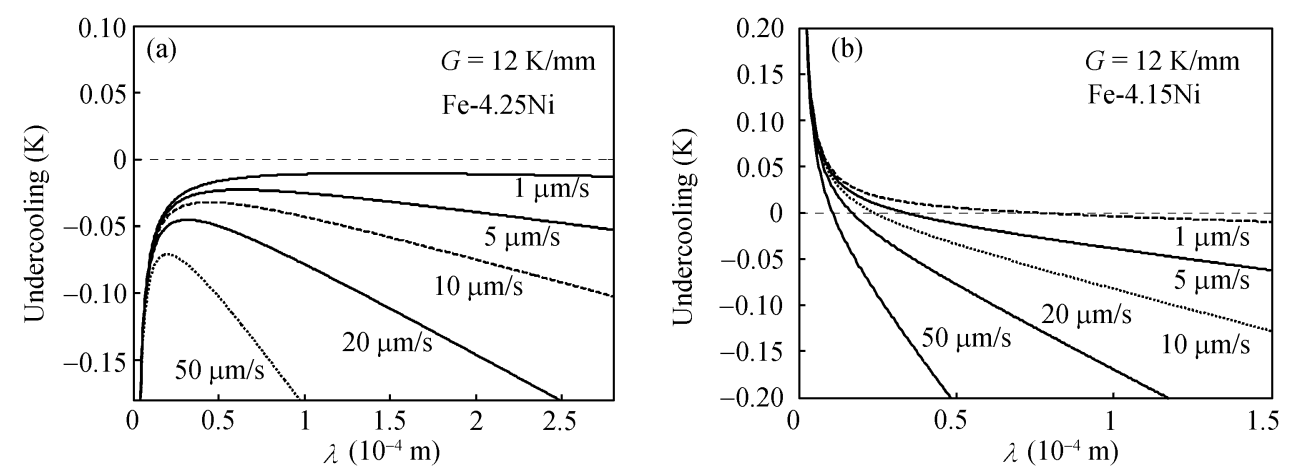

Figure 4 Influences of velocities on the $\Delta T-\lambda$ curves of $\mathrm{Fe}-\mathrm{Ni}$ coupled growth. (a) $\mathrm{Fe}-4.25 \mathrm{Ni}$; (b) $\mathrm{Fe}-4.15 \mathrm{Ni}$.

the most important is that the peritectic reaction cannot reach completion during PCG even if the growth rates are very low. Accordingly, the volume fractions of primary phase $\alpha$ are always larger than the values predicted using the equilibrium phase diagram. Obviously, this oversimplification can bring some errors to the calculation and even lead to erroneous conclusions. As a result, it is necessary to modify the previous model by taking into account the influences of the incompletion of the peritectic reaction.

\section{Peritectic coupled growth with peritectic reaction}

The main influence of the incompletion of the peritectic reaction on PCG is that it affects the volume fractions of two solid phases. Therefore, the key to modify the equilibrium peritectic coupled growth model is to take into account the influences of the peritectic reaction on the volume fractions of the two solid phases, especially on the primary phase $\alpha$ which dissolves during the peritectic reaction.

The equilibrium volume fraction of the primary phase in the equilibrium PCG model is obtained from the equilibrium phase diagram as

$$
f_{\alpha}=\frac{C_{\mathrm{p}}-C_{0}}{C_{\mathrm{p}}-C_{\alpha}}-\frac{C_{\mathrm{p}}-C_{\beta}}{C_{\mathrm{p}}-C_{\alpha}} \frac{C_{0}-C_{\alpha}}{C_{\beta}-C_{\alpha}} .
$$

In directional solidification, the peritectic coupled growth cannot reach completion during PCG. Unfortunately, it is difficult to quantitatively model the complicated dynamics of the influences of the peritectic reaction on the volume fraction of the primary phase during PCG. However, we can assume that only a portion of primary phase $\alpha$ can reach completion during the peritectic reaction, that is to say, the volume fraction of primary phase $\alpha$ that dissolves during the peritectic reaction is $\psi$. And then, the actual remanent volume fraction of $\alpha$ can be obtained as

$$
f_{\alpha}^{\prime}=\frac{C_{\mathrm{p}}-C_{0}}{C_{\mathrm{p}}-C_{\alpha}}-\psi \frac{C_{\mathrm{p}}-C_{\beta}}{C_{\mathrm{p}}-C_{\alpha}} \frac{C_{0}-C_{\alpha}}{C_{\beta}-C_{\alpha}} .
$$

Figure 5 shows the variation of the actual volume fractions of primary phase $\alpha$ with $\psi$. It is shown that the volume fractions of primary phase $\alpha$ can vary within a large region with $\psi$ increasing from 0 to 1 . Especially for hyperperitectic Fe-4.4Ni alloy, $f_{\alpha}^{\prime}$ can vary from 0 to 0.47 when $\psi$ increases from 0 to 1 ; whereas for the equilibrium peritectic coupled growth case, $f_{\alpha}$ is always zero, namely, the PCG is impossible. 


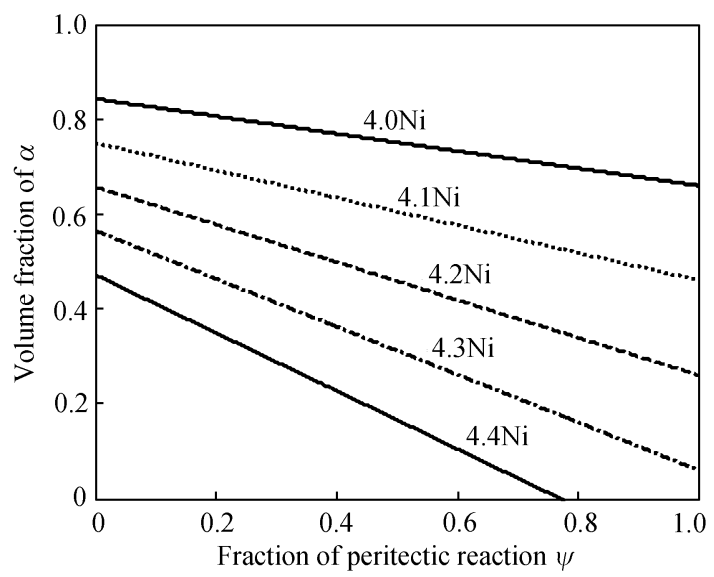

Figure 5 Variations of the volume fraction $f_{\alpha}^{\prime}$ of primary phase $\alpha$ with $\psi$.

Using eq. (17), the actual ratio of halfwidths of the two solid phases can be obtained as

$$
\xi^{\prime}=\frac{1-f_{\alpha}^{\prime}}{f_{\alpha}^{\prime}}
$$

Using $\xi^{\prime}$ from eq. (18) to replace $\xi$ in eq. (13), one can calculate the $\Delta T-\lambda$ curves with different peritectic reaction fraction $\psi$. Figure 6 shows the $\Delta T-\lambda$ curves with different peritectic reaction fractions as well as the experimental observations. It is shown that the observed undercooling is always smaller than the $\psi=100 \%$ curve, but well lies in the region from $\psi=60 \%$ to $\psi=$ $80 \%$. Furthermore, $a^{\mathrm{L}}$ in eq. (14c) may decrease from positive to zero and negative with the decrease of $\psi$, see the curves $\psi=62.3 \%$ and $\psi=60 \%$. It is interesting to find that the $\Delta T-\lambda$ curves are very close with increasing $\lambda$ when $\psi$ increases from $30 \%$ to $60 \%$. This may indicate that the coupled growths with different $\psi$ may reach the same state on the $\Delta T-\lambda$ curve at $a^{\mathrm{L}}=0$.

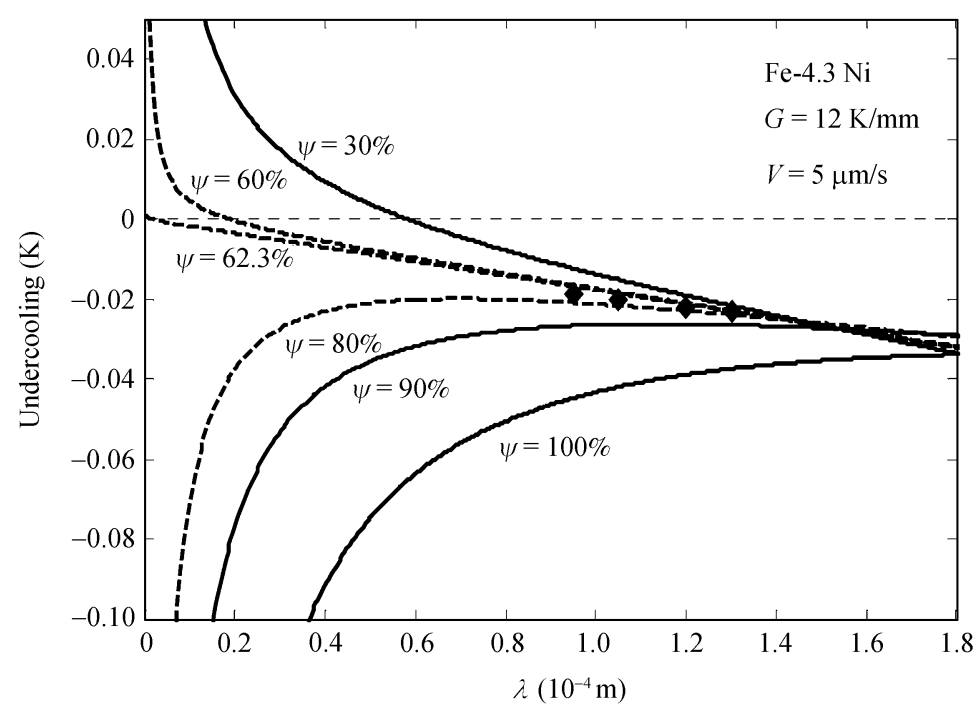

Figure 6 The $\Delta T-\lambda$ curves with different peritectic reaction fractions and the experimental results in Fe-Ni alloys represented as diamonds. 
It should be noted that the influences of the peritectic reaction on PCG are very complicated. Peritectic reaction around the trijunctions influences not only the volume fractions and diffusion fields, but also the morphological stability of $\mathrm{PCG}^{[17]}$, which needs more investigations in the future.

\section{Conclusions}

(1) The lamellar coupled growth of Fe-Ni alloy was analyzed using the equilibrium Boettinger-Jackson-Hunt model. It was shown that the interface status of Fe-Ni coupled growth can be well predicted by the previous model.

(2) The slope of the undercooling vs. lamellar spacing is very near zero around the minimum overheating, especially when $V$ is small. The coupled growth can exist under this condition even if the slope of $\Delta T-\lambda$ curve is slightly smaller than zero.

(3) The equilibrium peritectic coupled growth model was modified by considering the incompletion of the peritectic reaction. It was found that when the fractions of the peritectic reaction reach $60 \%-80 \%$ completion, the calculated $\Delta T-\lambda$ curves agree well with the experimental observations in the directionally solidified Fe-Ni alloys.

1 Jackson K A, Hunt J D. Lamellar and rod eutectic growth. Trans Met Soc AIME, 1966, 236: 1129-1142

2 Li S M, Ma B, Li X L, et al. Competitive growth of different phases in eutectic alloys under directional solidification. Sci China Ser E-Eng Mater Sci, 2005, 48(3): 270-281

3 Li J F, Zhou Y H. Effect of interface kinetics on the eutectic growth. Sci China Ser E-Eng Mater Sci, 2005, 48(4): 361 - 371

4 Orera V M, Merino R I, Pardo J A, et al. Microstructure and physical properties of some oxide eutectic composites processed by directional solidification. Acta Mater, 2000, 48(18-19): 4683-4689

5 Yang J M, Jeng S M, Bain K, et al. Microstructure and mechanical behavior of in-situ directional solidified NiAl/Cr (Mo) eutectic composite. Acta Mater, 1997, 45: 295-305

6 Kerr H W, Kurz W. Solidification of peritectic alloys. Int Mater Rev, 1996, 41(4): 129-164

7 Boettinger W J. The structure of directionally solidified two phase Sn-Cd peritectic alloys. Metall Trans A, 1974, 5: $2023-2031$

8 Busse P, Meissen F. Coupled growth of the properitectic $\alpha$-and the peritectic $\gamma$-phases in binary titanium aluminides. Scripta Mater, 1997, 36(6): 653-658

9 Lee J H, Verhoeven J D. Peritectic formation in the Ni-Al system. J Cryst Growth, 1994, 144(3): 353 - 366

10 Nagashio K, Takamura Y, Kuribayashi K. Coupled growth in the peritectic Nd-Ba-Cu-O system from highly undercooled melt. Scripta Mater, 1999, 41(11): 1161-1167

11 Vandyoussefi M, Kerr H W, Kurz W. Two-phase growth in peritectic Fe-Ni alloys. Acta Mater, 2000, 48(9): 2297-2306

12 Dobler S, Lo T S, Plapp M, et al. Peritectic coupled growth. Acta Mater, 2004, 52(9): 2795-2808

13 Kurz W, Fisher D J. Fundamentals of Solidification, Switzerland: Trans Pub Ltd, 1989

14 Dobler S, Kurz W. Phase and microstructure selection in peritectic alloys under high G-V ratio. Z Metallkd, 2004, 95(7): $592-595$

15 Vandyoussefi M, Kerr H W, Kurz W. Directional solidification and $\delta / \gamma$ solid state transformation in Fe-3\%Ni alloy. Acta Mater, 1997, 45(10): 4093-4105

16 Sumida M. Evolution of two phase microstructure in peritectic Fe-Ni alloy. J Alloys Compd, 2003, 349: 302-310

17 Su Y Q, Luo L S, Li X Z, et al. Well-aligned in situ composites in directionally solidified Fe-Ni peritectic system. Appl Phys Lett, 2006, 89: 231918

18 Fu H Z, Luo L S, Su Y Q, et al. Peritectic coupled growth in directional solidification of peritectic alloys. Chin J Nonferr Metal (in Chinese), 2007, 17(13): 349-359 